\title{
Un modelo de gestión de calidad total para las bibliotecas públicas
}

\author{
Mónica Izquierdo Alonso; Joaquín Ruiz Abellán \\ Grupo de Investigación SEMIODOC \\ Departamento de Biblioteconomía y Documentación \\ José Tomás Piñera Lucas. \\ Unidad de Evaluación \\ Universidad de Murcia \\ 30.071 Murcia
}

\subsection{Resumen}

En la primera parte del trabajo se presenta una serie de puntos introductorios donde se resalta: por una parte, las aportaciones de la perspectiva de la Gestión de la Calidad Total, en el campo de los servicios documentales; y por otra, se desarrolla, brevemente, el estado de la cuestión de la investigación realizada sobre este tema dentro de las Ciencias de la Documentación. La parte central del trabajo se dedica a asumir como hipótesis las actividades determinantes de la calidad total, fijar los objetivos y el método del modelo propuesto. Para concluir, se apuntan algunos resultados que exponen las actuales insuficiencias y problemas detectados en las bibliotecas públicas; y, como conclusión nuclear desprendida de nuestra investigación, se realza la figura del usuario: eje central de todo modelo de gestión de calidad total.

Palabras claves: Bibliotecas Públicas. Calidad Total. Servicios de Información. Gestión de Bibliotecas. Estudio de Usuarios. Evaluación Institucional. (Autor)

\subsection{Abstract}

In the first part of the work a series of introductory points are presented where it is stood out: on one hand, the contributions of the perspective of the Total Quality Management, in the field of the documentary services; and on the other hand, it develops, shortly, the state of the question of the investigation carried out on this topic within the Sciences of Documentation. The central part of the work is devoted to assuming the determinant activities of the total quality like hypot-

Scire. 2 : 1 (en.-jun. 1996). 
hesis, fix the objectives and the method of the nominative model. In order to conclude, some outputs are aimed that expose the current inadequacies and problems detected in public libraries; and, like nuclear conclusion removed of our investigation, the figure of the user is enhanced: central axis of all models of total quality management. (Author)

Keywords: Publics Libraries. Total Quality. Services of Information. Administration from Libraries. Study of Users. Institutional Evaluation.

\section{Introducción}

Las bibliotecas y, en general, todos los servicios de información-documentación han de mirar hacia adelante y adaptarse a los cambios que se están produciendo alrededor suyo, para responder a las necesidades de una sociedad que experimenta unas transformaciones continuas y bruscas. Una sociedad invadida por grandes masas de información que hacen que el ciudadano se sienta desorientado e incapaz de poder conseguir la información que le es necesaria, a pesar de la mayor especialización de los profesionales de la documentación y del avance de las nuevas tecnologías (con el desarrollo de las bases de datos, de las "autopistas de la información", etc.). Constatamos una preocupación generalizada por dotarnos de una gran cantidad de ofertas informativas, sin realizar previamente un estudio de la calidad de esa información. Por ello, frente a esa tendencia acumulativa, se pretende ahora que los nuevos servicios de información y documentación desarrollen programas de gestión de la calidad de los servicios y productos ofertados. Es decir, lo que se plantea es una nueva filosofía que haciendo compatibles los criterios cuantitativos y cualitativos, logre satisfacer las necesidades del usuario.

Es imprescindible recurrir a las aportaciones de la perspectiva de la Gestión de la Calidad Total (1) para adecuar nuestros servicios a las necesidades, tanto reales como potenciales, de los usuarios y poder mejorar los procesos de trabajo y control interno de nuestra organización bibliotecaria o servicio de información.

La calidad total es un concepto nacido en el ámbito de la empresa. Hoy los centros bibliotecarios y de documentación se encuentran ante el reto de cambiar los métodos de gestión tradicionales e introducir sistemas de calidad total en su organización y funcionamiento. Es necesario que los profesionales que trabajan en el ámbito de la información implementen sistemas de gestión de calidad total para adecuar sus servicios a esta nueva realidad.

Se ha de precisar que la calidad total dentro de éstos organismos públicos ha de ser considerada y tratada como un sistema. Por ello, resulta más apropiado hablar de Sistema de Calidad Total en los servicios bibliotecarios. Este sistema consta de los siguientes aspectos: una organización, unos procesos técnicos, unos

Scire. $2: 1$ (en.-jun. 1996). 
productos documentales, unos servicios y unos recursos necesarios para llevar a cabo la gestión de la calidad (Figura 1).

El diseño y desarrollo de un programa de calidad se inicia con la detección de las necesidades -implícitas y/o explícitas- de los usuarios de estos centros informativos; prosigue con el diseño del servicio a prestar y los productos que se han de ofertar; se complementa con la búsqueda de la optimización de los recursos financieros, humanos y técnico-tecnológicos; continua en el momento de la prestación del servicio, para volver a empezar de nuevo el programa cuando se mide el grado de satisfacción de los usuarios ante los productos y servicios prestados por la biblioteca. En caso de que surjan problemas, se buscarán las posibles correcciones o soluciones que solventen los fallos detectados. Para obtener un nivel alto de satisfacción de los usuarios se ha de intentar alcanzar unos grados aceptables de calidad en los distintos niveles que componen la organización bibliotecaria. De este modo, se logrará la calidad en: la dirección, los procedimientos, las relaciones internas entre personal de la biblioteca, las relaciones entre el personal bibliotecario y los usuarios, las relaciones con los proveedores, los servicios y productos ofertados al usuario y los recursos utilizados. El objetivo último es que todos estos niveles se interrelacionen estrechamente formando un sistema de calidad bibliotecario.

Tendremos en cuenta la calidad, si queremos ser competitivos y líderes en el suministro de servicios de información; y por ello las bibliotecas públicas han de desarrollar un programa de esas características. Al aplicar calidad, beneficiamos

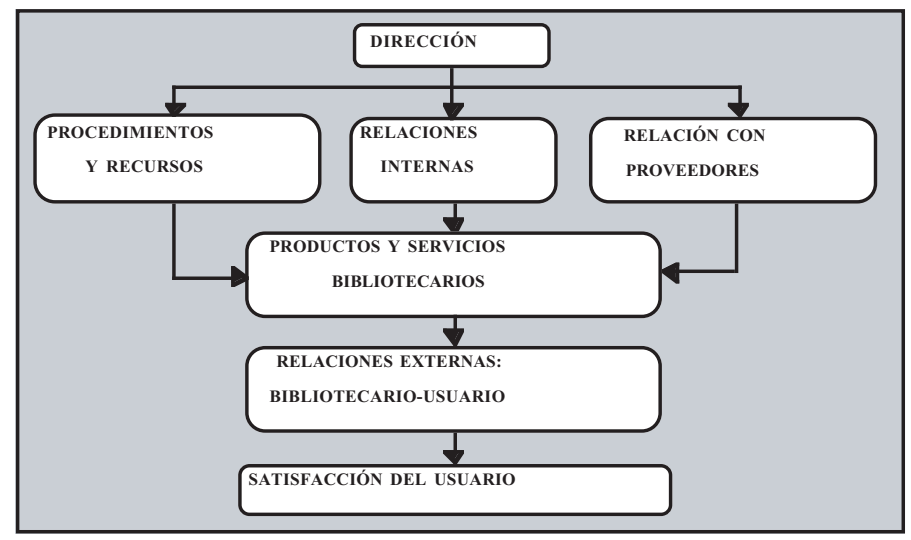

Figura 1. Diferentes niveles del sistema de calidad en la organización bibliotecaria

Scire. $2: 1$ (en.-jun. 1996). 
al usuario y hacemos que todos los integrantes de la organización se comprometan con la misión, objetivos y metas pretendidos por el centro. Además, la Gestión de la Calidad es fundamental para adaptar su grado de receptividad a las necesidades-demandas de los usuarios. Más en concreto, los principales objetivos por lograr con la implantación de un programa de Calidad Total en las Bibliotecas Públicas son los siguientes:

1. Disminuir costes, especialmente los atribuibles a los errores y desajustes organizativos.

2. Aumentar su competitividad dentro del sector público, garantizando su supervivencia.

3. Desarrollar un estilo de dirección centrado en el usuario, que fomente el trabajo en equipo y la autonomía del personal del centro bibliotecario.

4. Crear un clima de trabajo motivador y propicio para desarrollar la innovación y la creatividad.

5. Reestructurar la organización bibliotecaria, sus procedimientos de trabajo y actividades, a partir del objetivo estratégico de satisfacer a los usuarios.

\section{Origen y Desarrollo Histórico de los Estudios de Calidad en el Campo de los Servicios de Información}

El origen de los estudios de calidad en los servicios de información tiene un desarrollo reciente a partir de los estudios concernientes a la evaluación de estos servicios. El objetivo pretendido en todos los procesos de evaluación de dichos servicios es su eficacia, que no puede ser lograda más que a través de la satisfacción de los usuarios a los que se ofertan dichos servicios.

Es en los años sesenta cuando comienza el interés por la evaluación de los servicios de información. Más tarde, en los setenta, autores de gran relevancia en nuestro campo, como Lancaster (1977) y Saracevic (1977), comienzan a publicar y editar trabajos sobre el tema. Sin embargo, el gran precursor de la evaluación de los servicios de información fue Ernest De Prospo (1973); el primero, probablemente, que aborda el desarrollo de técnicas de evaluación de servicios aplicadas a distintos centros documentales. Los ingleses han contribuido, también de modo importante; cabe mencionar los trabajos de Totterdell y Bird(1976). En lengua francesa se han de mencionar los estudios de Eric Sutter(1992), que presentan análisis de la calidad total y publicidad en servicios de información,

Posteriormente, y bien avanzados los ochenta, los norteamericanos comienzan a modificar los planteamientos sobre evaluación de bibliotecas y servicios de información; siendo este un periodo caracterizado por una voluntad de poner en

Scire. $2: 1$ (en.-jun. 1996). 
práctica los métodos de evaluación de servicios de información, con herramientas fáciles de aplicar en el medio de trabajo y, si es posible, estandarizadas.

Es en las bibliotecas públicas donde los norteamericanos se han interesado más por aplicar sus estudios de evaluación. Ya en 1987 se publicó la segunda edición de la directrices o pautas normalizadas para la evaluación, que fueron desarrolladas a finales de los años setenta y después muy utilizadas en EE.UU. (Yan, 1987).

En España, la gestión de calidad total dentro del ámbito de las bibliotecas y servicios de documentación es de muy reciente introducción, aunque a mediados de los noventa se ha producido un crecimiento espectacular de experiencias de implantación de programas de calidad total. Destacan las aportaciones para distintos servicios de información de Duarte (1994) o Lloret Romero y Latorre Zacarés (1995).

\section{3. ¿Por qué es Importante la Calidad en el Campo de la Información?}

En una sociedad cambiante, donde todos los procesos varían a una velocidad vertiginosa, nuestras unidades de información no pueden quedar rezagadas, antes bien, deben correr en paralelo, y muchas veces anticiparse, a los futuros requerimientos del entorno. La información ha llegado a ser cada vez más un bien estratégico para organismos o instituciones de todo tipo. Ello significa que el personal de dichos organismos -la clientela de los servicios de información- va a ser cada vez más exigente en cuanto toque a la eficacia del servicio suministrado y la buena adecuación de los productos de información a sus necesidades crecientes.

El crecimiento casi exponencial de las fuentes de información mundial y la multiplicación de los soportes han hecho necesario el desarrollo de tecnologías que permitan obtener informaciones fiables, así como una gestión moderna y dinámica de los dispositivos de tratamiento y de transferencia de información que implique, como en el caso de una empresa, la introducción de nuevos métodos de trabajo, la puesta en marcha de nuevos productos, y una orientación "empresarial" de la actividad documental-bibliotecaria.

Como en otras actividades económicas, las actividades documentales no evolucionarán al mismo ritmo que la sociedad, si no se emplean métodos de gestión moderna y se aplican, como parte sustancial de estos métodos, programas de gestión de calidad total. Es hora de introducir la calidad en los productos y servicios de información y documentación. Concretando, los puntos básicos de la Calidad Total en el sector de la información son tres:

1. La calidad del producto documental que permite satisfacer las expectativas del usuario con el menor costo.

Scire. 2 : 1 (en.-jun. 1996). 
2. La calidad de producción y de sus procedimientos de control.

3. La calidad del servicio que acompaña al producto.

La intuición y la improvisación han dejado de ser los motores de la gestión. En su lugar, la aplicación de nuevas técnicas de gestión nos va a ofrecer un conocimiento más exhaustivo de nuestro entorno y va a ser el eje central no sólo del desarrollo de nuestros centros, sino también de la supervivencia de los mismos.

Los procesos de un servicio de información -biblioteca o centro de documentación- son, generalmente, complejos: en todos ellos intervienen personas distintas llevando a cabo operaciones diversas. Así, por ejemplo, para poner un documento a disposición del usuario, se debe seguir un proceso que puede implicar a varias personas: desde su selección y adquisición por el centro, pasando por los distintos departamentos de proceso técnico, hasta la recepción del documento por el usuario final.

En el éxito de este proceso concurren factores muy diversos: el nivel de agilidad del sistema para tramitar las compras, los plazos de entrega del proveedor, la realización de las tareas y procedimientos en el procesamiento técnico del documento, el sistema de descripción utilizado, la descripción propiamente tal (campos que permitirán el acceso al documento), la forma en la que se colocan los documentos en las estanterías con el fin de facilitar su localización, etc. Por tanto, una simple petición o consulta de un usuario se convierte para el centro en un conjunto complejo de acciones; aunque sean percibidas por el usuario como un todo indivisible. Y la calidad que el usuario percibe no es la calidad de las partes, sino la calidad del conjunto del servicio recibido. La calidad total es, pues, la calidad en todos y cada uno de los elementos que intervienen en un servicio y, en particular, de todas las personas que intervienen en su prestación, desde el director al conserje.

\section{Modelo de Gestión de la Calidad Total para las Bibliotecas Públicas}

Contando con los constituyentes del resúmen -asimismo, indicadores básicos del tratado científico-(2) presentaremos ya el modelo de gestión de nuestra propuesta. Tras asumir como hipótesis las actividades determinantes de la calidad total (4.1), fijamos los objetivos (4.2) y el método (4.3); a modo de resultados (4.4), a la luz de estos propósitos, constatamos las actuales insuficiencias y problemas detectados en las bibliotecas públicas; y, como conclusión nuclear (4.5) desprendida de nuestra investigación, realzaremos la figura del usuario: eje central de todo modelo de gestión de calidad total.

Scire. 2 : 1 (en.-jun. 1996). 


\subsection{Consideraciones previas}

El modelo básico de implantación de un sistema de gestión de calidad total en una biblioteca está determinado por cuatro grupos de actividades:

- Adquisición: aglutina las actividades relacionadas con la adquisición de documentos. La biblioteca actúa como usuario.

- Proceso técnico: agrupa las actividades que tienen que ver con la disponibilidad de los documentos y la puesta en disposición del usuario.

- Difusión de información: reúne las actividades relacionadas con las actividades de los servicios de información, referencia, extensión cultural, etc. en las que la biblioteca actúa como proveedor.

- Evaluación, a tres niveles: del sistema, de los servicios, y de los procesos.

Además, para el desarrollo de un modelo de calidad total en una biblioteca pública es necesario un análisis que incluya: la revisión de las condiciones y servicios existentes; la delimitación de objetivos; la determinación y aplicación de un sistema de estrategias para alcanzar dichos objetivos; y, por último, la evaluación periódica de este proceso (Cuadro 1).

\subsection{Objetivos generales del modelo de calidad propuesto}

Partiendo de estas consideraciones, y como concreción especial del apartado

1. Revisión de las condiciones y servicios existentes:

1.1.Descripción de la situación actual de la biblioteca.

1.2.Estudio y valoración de los procesos de cada uno de los servicios bibliotecarios.

2. Delimitación de objetivos generales y específicos para cada uno de los servicios o áreas a analizar.

3. Determinación y aplicación de un sistema de estrategias para alcanzar dichos objetivos:

3.1.Grado de inmersión o análisis comparativo del centro con su entorno, su inserción e irradiación en la población, su nivel de promoción social, etc.

3.2.Grado de pertinencia o identificación de oportunidades, fortalezas, debilidades y amenazas.

3.3.Finalidad o estudio de los medios necesarios para alcanzar los objetivos previstos.

4. Evaluación periódica de este proceso. Estudios casuísticos.

Cuadro 1. Un Modelo de Calidad para una biblioteca: aspectos del análisis

Scire. $2: 1$ (en.-jun. 1996). 
segundo del análisis -registrado en el Cuadro 1: Un modelo de calidad para una biblioteca: aspectos del análisis- el modelo de calidad que proponemos apunta hacia los siguientes objetivos generales:

1. Diseñar la biblioteca pública del siglo XXI, bajo las directrices de la filosofía de la calidad total, de modo que permita obtener un servicio moderno, competitivo, acorde a las nuevas necesidades de la comunidad y que esté dispuesto a adelantarse a las expectativas futuras de sus usuarios. Se pretende lograr unos centros públicos activos, rentables, regidos bajo parámetros similares a los de cualquier empresa puntera y líder en su actividad; sin que llegue a perderse su dimensión social y cultural de carácter universal. En definitiva, se intenta conseguir un biblioteca que pueda llegar a convertirse en un centro público con mecanismos de autofinanciación que le permitan sostener sus prestaciones y servicios y poder mejorarlos continuamente. Pues todos somos conscientes de las limitaciones y carencias del presupuesto público, que hacen que muchas bibliotecas públicas vean limitadas sus funciones y servicios. Es necesario un cambio en la organización interna del centro y presumiblemente en la legislación oficial.

2. Aproximar la Biblioteca a las necesidades y peticiones de sus usuarios reales o potenciales. Para optimizar la difusión de la información, las bibliotecas tienen que conocer a fondo a sus usuarios: quiénes y cuántos son, y qué necesitan. El primer punto de interés primordial para nuestros centros es el conocimiento del usuario, es decir, su análisis; extendiendo el término "usuario" tanto a los ciudadanos que conocen y utilizan el servicio como aquellos que no lo hacen. A este respecto, la IFLA es rotunda cuando dice: "la aplicación de los medios debería basarse en una apreciación de las necesidades (servicios bibliotecarios) de la población local en su conjunto y no sólo en las demandas de los actuales usuarios de la biblioteca" (FIAB, 1988). En definitiva, el término "usuario" designa a: "todos los beneficiarios en potencia y no sólo a los que utilizan los servicios en un momento determinado".

3. Valorar las instalaciones, los equipos técnico-tecnológicos, los materiales empleados en los procesos de trabajo, etc. para poder lograr el máximo rendimiento de todos estos recursos materiales.

4. Conocer todos los aspectos de los recursos humanos de que disponemos, para lograr un equipo de trabajo concienciado y motivado que ponga en marcha un servicio bibliotecario enmarcado dentro de la filosofía de gestión de calidad total. Es vital, para obtener éxito en el programa de calidad, que todos los miembros de la organización se sientan integrados en el proyecto y sepan apreciar que el trabajo individual bien hecho y armonizado con el plan y los objetivos generales de la biblioteca conduce a la mejora de todo el sistema de la institución a la que pertenecen.

Scire. 2 : 1 (en.-jun. 1996). 
5. Desarrollar planes de autofinanciación y reestructuración de los gastos.

6. Facilitar la disponibilidad de los fondos documentales: que cuando un usuario busque un documento concreto, pueda encontrarlo con facilidad y el mínimo de ayuda, y consultarlo rápidamente.

7. Posibilitar el acceso preciso y puntual a la información: que cuando un usuario quiera encontrar documentación sobre algo en concreto, pueda saber si la tenemos, escoger la que le interese, consultarla y, en caso de no tenerla, saber dónde y cómo la puede encontrar.

8. Conseguir un crecimiento equilibrado del fondo documental: que las adquisiciones vayan dirigidas a complementar perfiles y que se obtenga la máxima rentabilidad del presupuesto.

9. Potenciar la difusión: que los posibles usuarios conozcan la biblioteca, sus fondos y los servicios que se les ofrecen. Se hacen necesarias dentro de las bibliotecas públicas políticas de marketing y de estudio de mercado.

10. Realizar las reorientaciones necesarias para el buen funcionamiento de los servicios.

11. Romper el habitual "encapsulamiento" que vienen padeciendo nuestras bibliotecas y realizar el descubrimiento del "mundo exterior", obligándolas a mantener un diálogo continuo con su entorno.

12. Flexiblizar las estructuras, a fin de facilitar al usuario la accesibilidad (fondos,horarios, préstamo, información, etc.).

13. Realizar una cambio de imagen, tanto de centro como de personal.

14. Evolucionar sincrónicamente con el entorno. Para ello, los centros deberán dotarse de métodos de control que reflejen fielmente las variaciones que se produzcan internamente en su organización y en el uso de sus servicios, con el objeto de conseguir una valoración del servicio total ofertado.

La definición de estos objetivos tiene en cuenta los recursos humanos y materiales disponibles. Al mismo tiempo, son objetivos mensurables cuantitativa y cualitativamente. Es necesario temporalizar, generalmente por trimestres o por cursos. El conjunto de todos los objetivos dentro de cada área clave de servicio, con su temporalización, tipos y responsables, nos da como resultado el plan de trabajo.

\subsection{Metodología}

El Proyecto de Calidad de la Biblioteca Pública se ha de planificar sobre la dinámica de la gestión de la calidad total. El elemento inicial del proyecto debe ser la definición de una nueva estructura organizativa y la reorganización de todos los servicios. Se pretende iniciar un conjunto de estrategias específicas

Scire. 2 : 1 (en.-jun. 1996). 
sobre la perspectiva organizativa y la mejor forma de facilitar el cambio. Para ello, hemos de partir de la consideración de que es posible aumentar la efectividad estableciendo canales de comunicación abiertos para responder a las necesidades del equipo de dirección. Tomando como base una adaptación de los elementos que componen el Modelo de Gestión de Calidad Total: satisfacción al usuario, satisfacción al personal bibliotecario, estilo de dirección, gestión del personal, recursos, procesos, impacto social y resultados, se determinarán las áreas prioritarias que sirven de marco a nuestro modelo propuesto: unidad dentro de la diversidad de la biblioteca (aplicación de la teoría de sistemas), innovación e iniciativa, participación del personal, estructura organizativa, diagnóstico, objetivos, acciones de mejora, gestión de procesos, comunicación abierta y control. Asimismo, se plantearán cuatro líneas de acción: reorganización de la estructura organizativa, hacia la adopción de la Gestión de la Calidad Total, aplicación de las directrices de las normas ISO 9000 a los servicios de proceso técnico -adquisiciones, proceso bibliográfico, etc.- y a los servicios de información y referencia.

De forma más explícita, señalamos la metodología propuesta para nuestro modelo de Calidad Total, que queda recogida en los siguientes puntos:

1. Reunión inicial con el director de la biblioteca, para explicar el programa de calidad que se desea poner en marcha. Se aprovechará este primer contacto con el centro para poder recabar información sobre las funciones que se desarrollan, servicios y productos ofertados, estructuración y organigrama, personal, instalaciones, problemas que se han detectado, proyectos futuros, etc.

2. Se realizará una sesión informativa, dirigida al personal de la biblioteca, sobre qué es un programa de gestión de calidad y lo que supone aplicarlo a un servicio público como es la biblioteca. Se darán todo tipo de aclaraciones y se resolverán las dudas que puedan surgir al respecto. El fin de esta reunión es la motivación y participación de todos los empleados para que éstos se sientan implicados y protagonistas en el proyecto. Se analizarán, además, todos los aspectos de la organización, discutiéndose los fallos o defectos detectados e intentando buscar sus causas y posibles soluciones.

3. Se realizarán varias visitas a la biblioteca durante días y horas distintos de la semana, diferenciando las horas de atención al público y las destinadas a trabajo interno. También, se efectuará un análisis por cada una de las áreas o servicios que integran el centro bibliotecario. Este análisis nos permitirá delimitar objetivos específicos y planes de actuación en cada una de las áreas.Toda esta información constituirá una primera valoración sobre la situación actual de la biblioteca; que nos posibilitará la formulación de una serie de prioridades y de

Scire. 2 : 1 (en.-jun. 1996). 
líneas de actuación, que formarán parte del esquema inicial de nuestra planificación.

4. Elaboración de la planificación estratégica del proyecto de calidad. No olvidarnos la primera regla de la calidad: que toda la actividad a desarrollar ha de presentarse por escrito o estar recogida en un manual de organización y procedimiento que contenga todos los pasos del plan de trabajo que se van a poner en marcha.

5. Nueva sesión informativa con el personal de la biblioteca en la que se expondrán los puntos que conforman el proyecto diseñado para la planificación estratégica del centro. Además, se elegirá a un miembro de cada sección de la biblioteca para formar un comité de calidad que se reunirá de forma más asidua para ir controlando y modificando, si se da el caso, la efectividad de la planificación una vez puesta en práctica.

6. Diseño de encuestas por muestreo y cuestionarios. Para ello, se ha de seguir una serie de etapas: primero una etapa de planificación, que incluye el establecimiento de las metas de la encuesta y la creación de la estrategia general para obtener y analizar los datos. Una segunda etapa de elaboración del diseño de la muestra, en los casos en los que vaya a ser necesaria su utilización, y elaboración de los cuestionarios y encuestas. Es ésta una fase en la que se han de trasladar los amplios objetivos del estudio a preguntas que logren obtener la información necesaria. Es un proceso típico de tanteo que implica largas horas de discusión y numerosas horas previas. El mayor esfuerzo se focaliza a la hora de establecer el número y tipo de las preguntas, su secuencia y los medios para motivar a la persona encuestada y mantener su interés. La tercera etapa es la de edición y codificación; procesos para convertir las preguntas registradas en el cuestionario a categorías, por lo general expresadas numéricamente, que puedan ser contabilizadas o tabuladas.

A continuación, apuntaremos algunos recursos para la compilación de datos; recursos que se utilizan para obtener y registrar datos originales. Si se quieren obtener datos válidos y exactos se ha de tener un cuidado extremo a la hora de diseñarlos y usarlos.

6.1. Cuestionarios escritos. Un cuestionario bien fundado habrá de ser cuidadosamente diseñado y estar basado en un determinado propósito y objetivo específico. Esto requiere algo más que formular unas cuantas preguntas conversacionales. Observaremos las siguientes especificaciones:

- Formular preguntas simples y cortas.

- Formular preguntas de una sola respuesta. No se deben combinar dos preguntas en una.

Scire. 2 : 1 (en.-jun. 1996). 
- Evitar palabras o preguntas de connotación afectiva.

- Utilizar preguntas neutrales que eviten formulaciones intencionadas; que suelen originar desviaciones.

- No utilizar dobles negaciones.

- Permitir toda la gama de respuestas. Por ello se han de evitar las preguntas de respuestas limitadas.

- Especificar, en su caso, si la respuesta a una pregunta depende de la respuesta a otra pregunta.

Todos los cuestionarios deberían ser probados con anterioridad y revisados, de modo que no contengan errores obvios: ambigüedades, malas interpretaciones, palabras equivocadas, interpretaciones conflictivas, alta tasa sin respuesta, etc.

6.2. Encuestas orales con una serie de preguntas. Las entrevistas cara a cara con una serie de preguntas se usan a menudo para llevar a cabo estudios de mercado. Lo que se refirió con anterioridad en los cuestionarios también se aplica a esta situación. El verdadero problema en las entrevistas de masas es el muestreo.

6.3. Entrevistas telefónicas. Lo que se detalló sobre el uso y el diseño de los cuestionarios escritos también es aplicable en este caso.

6.4. Escalas de valoración. Las escalas de valoración se usan para obtener información sobre la calidad de los servicios. Las valoraciones más comunes son: malo, regular, bueno y excelente. Algunas veces incluyen alguna otra calificación. Sin embargo, las escalas de valoración tienen algunos puntos débiles con respecto a una información útil para los propósitos del control de calidad. Así, por ejemplo: no aparecen las actividades y condiciones específicas en las que se basan las valoraciones; una misma valoración dada por clientes distintos puede representar muchas actividades y cumplimientos diferentes; lo que les gusta a unas personas a otras les disgusta, y así el mismo servicio puede recibir una amplia gama de valoraciones. Por último, se ha de señalar que las valoraciones basadas en experiencias diferentes no son comparables y, por tanto, no pueden sumarse ni cuantificarse de manera significativa alguna. Lo que se necesita no es una escala de valoraciones, sino un informe del servicio.

El modelo de gestión de calidad de la biblioteca, entendiendo ésta como un sistema, nos llevará a un análisis de cada una de las partes constituyentes de dicho sistema. Comenzaremos por describir la unidad de información, ya existente: su naturaleza, ubicación, recursos materiales, económicos y humanos, procesos y productos que ofrece el centro; para obtener un diagnóstico de estado del centro. Posteriormente efectuaremos un estudio de usuarios que nos permita definir -en su caso redefinir- y orientar los productos y servicios ofrecidos por la biblioteca.

Scire. $2: 1$ (en.-jun. 1996). 
Dicho estudio requerirá, en primer lugar, una identificación del tipo de usuarios al que responde nuestra unidad de información y, posteriormente, una evaluación de sus necesidades informativas. Éstas pueden ser de varios tipos: puesta al día en el campo de interés y temas afines, información requerida para llevar a cabo el trabajo cotidiano, búsquedas retrospectivas en caso de investigaciones, etc. El análisis nos permitirá la determinación del contenido y tipo de información que busca el usuario, desde un punto de vista cuantitativo y cualitativo.

7. En la etapa de recogida de información sobre los puntos fuertes y débiles de la biblioteca se distinguirán dos niveles:

7.a. Un primer nivel, destinado al análisis de la estructura interna del centro: instalaciones, plantilla de personal, procesos de trabajo, rendimientos, horarios, estructuración de las áreas o servicios, funcionamiento de cada una de ellas, etc. Para ello, se distribuirá entre el personal del centro una serie de cuestionarios. Esta información se complementará y contrastará con las respuestas de un segundo cuestionario realizado a los usuarios del centro. Dentro de este primer nivel distinguimos 6 bloques de cuestionarios:

Primer bloque: Evaluación general de la biblioteca. Se realizarán preguntas alusivas a: la ubicación del centro, dimensiones, acceso, espacios, condiciones de las instalaciones, medidas de seguridad, composición de la plantilla del personal de la biblioteca, los servicios que se prestan en la biblioteca, el equipamiento o materiales con los que cuenta el centro, haciéndose especial hincapié en la incorporación de nuevas tecnologías, y el presupuesto con el que cuenta la biblioteca para el desarrollo de sus actividades. Este primer bloque nos aproxima a la realidad de la biblioteca y permite conocer con qué recursos se cuenta y cuál es el funcionamiento del centro. Asimismo, con esta información podremos averiguar cuál es el punto de partida de nuestro análisis y comprender mejor la situación que atraviesa el centro bibliotecario que estamos analizando. No olvidemos, que para poder prestar un servicio de calidad es necesario disponer de unas instalaciones, un personal, unos materiales, etc. que nos permitan prestar un servicio óptimo, que satisfaga por completo las necesidades de nuestros usuarios. Los usuarios deben sentirse cómodos con las instalaciones, el personal, los materiales que utilizan. Debe de contar también con unos servicios y productos documentales de calidad. En definitiva, el usuario tiene que identificarse con los objetivos y fines últimos de la biblioteca pública. Como la voz del usuario es fundamental, en el segundo nivel de nuestro estudio consideramos necesario el diseño de un segundo bloque de cuestionarios en los que el usuario valore todos estos aspectos y, de esta forma, podremos contrastarlos con los datos obtenidos en este primer cuestionario en el que ha respondido el personal interno de la biblioteca.

Scire. 2 : 1 (en.-jun. 1996). 
Segundo bloque: Cuestionarios de análisis de procesos y evaluación de tareas o actividades llevadas a cabo en los distintos departamentos de la biblioteca (adquisición y selección de fondos, catalogación, préstamo, información bibliográfica y referencia, extensión cultural, etc.). La evaluación de cada uno de los servicios que integran la biblioteca tiene como objetivos fundamentales: A) Proporcionar una visión más completa de cómo estos servicios utilizan los recursos disponibles y hasta qué punto atienden a las necesidades de la comunidad a la que sirven. B) Analizar los puntos débiles y fuertes de cada uno de los servicios de la biblioteca. C) Averiguar las modificaciones posibles para mejorar el rendimiento de estos servicios. D) Evaluar el nivel general de satisfacción con los servicios prestados por la biblioteca. E) Determinar cuáles y cómo deberían ser los servicios que los usuarios desearían que fuesen prestados en la biblioteca.

Tercer bloque: Evaluación de equipos de trabajo. Conocer y evaluar el comportamiento y la dinámica de los equipos de trabajo dentro la biblioteca permite: adquirir estrategias para resolver los conflictos grupales, conocer mejor la creación de ideas del personal de la biblioteca, poder desarrollar acciones e inculcar un sentimiento del "nosotros" que nos permita crear un servicio bibliotecario, en conjunto, más eficaz y acorde con la filosofía de la gestión de la calidad total. Los objetivos de este tercer bloque son: A) Reflexionar sobre el comportamiento y dinámica de los equipos de trabajo. B) Saber evaluar un grupo. C) Observar el nivel de cooperación y el interés por trabajar en equipo.

Cuarto bloque: Evaluación de las reuniones de trabajo. Junto al trabajo en grupo, es importante desarrollar dentro de la biblioteca una dinámica de cooperación y entrega de todos los miembros para poder implementar un programa integral de calidad. La reunión es el instrumento esencial por el que cada uno de los integrantes de los diversos departamentos de la biblioteca puede aportar ideas innovadoras, soluciones a los problemas, etc., posibilitando de este modo, que todos los miembros de la organización se vean involucrados e identificados con el programa de calidad. Los objetivos de este cuarto bloque son: A) Controlar el desarrollo de las reuniones. B) Analizar y valorar las reuniones. C) Concienciar al personal de la biblioteca de la importancia que tienen las reuniones de trabajo para aportar ideas creativas a proyectos y buscar soluciones a todo tipo de problemas.

Quinto bloque: Evaluación del estilo de dirección. En todo programa de calidad debe de haber una cabeza visible encargada de: dirigir, supervisar, coordinar, gestionar, planificar, asignar, documentar, ejecutar, comunicar, motivar y delegar todas las tareas necesarias para poner en marcha el programa de calidad. La figura del líder ha de poseer una madurez técnica que le permita conocer perfectamente su área de trabajo, que le posibilite una capacidad destacada para resolver problemas y asumir responsabilidades y la entereza para desarrollar un pro-

Scire. 2 : 1 (en.-jun. 1996). 
grama donde se lleven a cabo todas sus fases y se cumplan a la perfección los plazos asignados a cada una de ellas. Junto a ésta ha de poseer también una madurez psicológica marcada por la constancia en sus tareas, la autonomía, la motivación hacia la obtención de resultados, la voluntad, una disposición para asumir responsabilidades y la actitud positiva y constructiva hacia su trabajo. Blake y Mouton (1964) inventaron una representación gráfica de la concepción del liderazgo, proponiendo una cuadrícula gerencial basada en los estilos de "interés por la gente" e "interés por la producción". Los objetivos pretendidos son: A) Averiguar el estilo personal del liderazgo. B) Aproximarnos a las funciones directivas.

Sexto bloque: Diagnósticos de puestos de trabajo. La finalidad de este bloque es establecer cómo pueden ser diseñados mejor los puestos de trabajo por medio de la obtención de reacciones individuales a diferentes clases de puestos de trabajo. Se analizará la percepción de la persona respecto a su puesto de trabajo y sus reacciones para con él. Así, se proporcionará información relativa a la valoración, por quién ocupa el puesto de trabajo, de su situación actual de trabajo y sus preferencias, en caso de que el trabajo llegase a ser reorganizado. También, permitirá una información individual más amplia, de modo que sea posible evaluar con generalidad la satisfacción con el entorno de trabajo o con el contexto del propio trabajo, la satisfacción relacionada con el trabajo en factores específicos y la fuerza de las necesidades de crecimiento. En definitiva, se proporcionará la base para recoger los datos básicos para los planteamientos de los diseños de trabajo. A través de ello, es posible juzgar el grado de satisfacción de la persona con características del contexto de trabajo, del propio trabajo, y con las preferencias personales. La información finalmente obtenida nos permitirá organizar la plantilla para poder desempeñar de un modo eficiente cada una de las tareas asignadas, contribuyendo así al programa de calidad. Para concluir, podemos señalar los objetivos que se pretenden en este bloque: A) Establecer cómo pueden ser diseñados mejor los puestos de trabajo. B) Valorar la satisfacción del empleado con respecto a su trabajo.

7.b. En un segundo nivel, se efectuará un estudio de usuarios. No olvidemos al respecto que se partirá de una información previa obtenida en el nivel anterior, pero que ha de ser comparada y ampliada en esta segunda fase. Aquí se valorarán tanto los servicios como los productos ofertados por el centro comprobando si éstos responden a las necesidades tanto reales como potenciales de los usuarios. Se analizará también qué nivel de promoción tiene el centro, su proyección en el exterior, su consideración social, etc. Dentro de este nivel se han distinguido cinco bloques para diseñar los cuestionarios:

Primer bloque: Encuesta referente a la impresión general de los usuarios tras efectuar una visita al centro. Los objetivos de esta encuesta son: A)

Scire. 2 : 1 (en.-jun. 1996). 
Determinar quién utiliza la biblioteca y para qué. B) Evaluar si los usuarios obtienen lo que desean en sus visitas a la biblioteca. C) Acopiar información sobre las opiniones de los usuarios o la percepción sobre los servicios que presta la biblioteca.

Segundo bloque: Encuesta de opinión de los usuarios de la Biblioteca Pública. Los objetivos últimos de esta encuesta son los siguientes: A) Determinar quién utiliza la biblioteca y para qué. B) Evaluar el nivel general de satisfacción de los usuarios con los servicios prestados por la biblioteca. C) Determinar qué servicios desean fundamentalmente los usuarios que preste la biblioteca.

Tercer bloque: Encuesta de actitud de los usuarios. El objetivo de esta encuesta es evaluar las actitudes de los usuarios y de los no usuarios con respecto a la biblioteca.

Cuarto bloque: Cuestionario sobre aspectos generales y naturaleza de la biblioteca, desde el punto de vista del usuario. El objetivo de este cuestionario es averiguar qué opinión tiene el usuario del centro sobre cuestiones tales como: la ubicación del centro, dimensiones, acceso, espacios, condiciones de las instalaciones, medidas de seguridad, composición de la plantilla del personal de la biblioteca, los servicios que se prestan en la biblioteca, el equipamiento o materiales con los que cuenta el centro, haciéndose especial hincapié en la incorporación de nuevas tecnologías, etc. Como se apuntó anteriormente esta encuesta debe ser contrastada con el cuestionario desarrollado en el primer bloque de nuestro primer nivel de estudio.

Quinto bloque: Formulario de evaluación para un usuario de un servicio de búsqueda en línea. El objetivo último de la evaluación de los servicios en línea es: A) Descubrir y analizar los casos con resultados pobres e insatisfactorios por parte de los usuarios. B) Averiguar las modificaciones posibles para mejorar el rendimiento de los servicios en línea.

8. Entrevista con el director de la biblioteca y el personal bibliotecario. En esta fase se les distribuirá una serie de cuestionarios, se realizarán una serie de entrevistas estructuradas, etc. que nos lleven a hacer un primer diagnóstico de la realidad del centro atendiendo a infraestructura, recursos financieros y humanos, procesos técnicos, productos ofrecidos por el centro, tipología de usuarios, tecnología utilizada, secciones o áreas en las que se estructura la biblioteca, etc.

9. Realización, en varias sesiones, de entrevistas y encuestas a una muestra significativa de usuarios del centro. En una segundo nivel, se pasará un cuestionario a estos usuarios para que evalúen de forma precisa los servicios y productos ofertados por el centro y de este modo, poder ver si éstos se adecuan a sus necesidades y demandas. Previamente a esta fase, se habrá hecho una investigación de mercado sobre los usuarios que acceden a este centro; se habrán definido

Scire. 2 : 1 (en.-jun. 1996). 
los productos generados, sus componentes y procesos técnicos, su personal y la organización de la producción y servicios.

10. Recolección y tabulación de los datos obtenidos en los distintos muestreos y cuestionarios, y análisis y elaboración del informe final, consistente en la presentación e interpretación de los datos. La fase de preparación para el análisis cubrirá una variedad de tareas, como: asignación de diferentes ponderaciones, establecimiento de escalas y variables compuestas, tabulación estadística, etc.

11. Con los datos obtenidos en las fases anteriores se obtiene un diagnóstico que nos refleja los puntos fuertes y débiles que presenta el centro. Pero lo que realmente nos interesa de este diagnóstico es detectar los fallos y seleccionar las áreas o servicios a mejorar para poder poner en práctica medidas correctivas que solucionen y mejoren las debilidades detectadas. Dichos datos nos permitirán la identificación de una serie de indicadores(3) que posibilitarán la evaluación de los resultados obtenidos en el proceso de calidad. Los indicadores han de reunir unas características:

1. Apropiados al tamaño de la organización o a las posibilidades técnicas de recogida de información.

2. Susceptibles de indicar problemas operativos y sus eventuales soluciones.

3. Válidos para permitir establecer objetivamente la relación entre el objeto medido y el resultado obtenido, tanto por bibliotecarios como por no bibliotecarios.

4. Reproducibles, a fin de permitir la estimación de su grado de confianza.

5. Comparables, de tal manera que permitan la identificación de los factores determinantes de una situación particular.

6. Prácticos e integrables en procedimientos rutinarios.

7. Creíbles para los usuarios de la evaluación.

Para poder realizar un diagnóstico de una biblioteca hemos de utilizar unos Indicadores básicos: Evolución de las colecciones. Número anual de documentos adquiridos. Presupuesto de adquisiciones. Número de suscripciones. Plantilla total. Títulos catalogados. Ejemplares prestados/consultados. Gastos totales. Dinamismo/estancamiento de la biblioteca. Eficacia de los servicios técnicos. Eficacia de los servicios públicos. Tasa de actividad. Tasa de rendimiento. Tasa de satisfacción del usuario. Basándonos en lo que se ha señalado en el punto 4.1., en torno al modelo básico de implantación de un sistema de gestión de calidad total en una biblioteca y su estructuración en grupos de actividades, hemos diferenciado cinco grupos de indicadores básicos (4): 
Grupo 1: Adquisiciones:

- Plantilla

- Tiempo de disponibilidad de los documentos

- Novedades

- Cobertura de las colecciones

- Peticiones satisfechas

- Peticiones no satisfechas

- Tiempos de entrega del material

- Reclamaciones

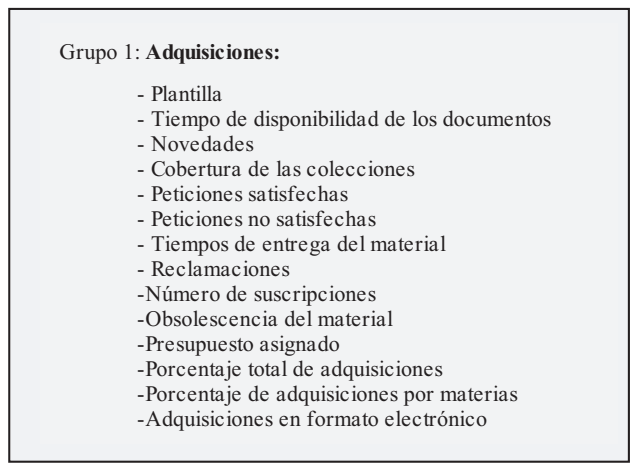

\begin{tabular}{|l|}
\hline Grupo 2: Proceso técnico: \\
- Plantilla \\
- Catalogación/ Clasificación original \\
- Catalogación/Clasificación delegada \\
- Procesado \\
- Disponibilidad para el usuario \\
- Disponibilidad de circulación \\
- Control de calidad de registros \\
- Automatización de procesos \\
- Tiempo \\
- Coste de los procesos \\
- Productos elaborados \\
- Catálogo en línea (OPAC) \\
- Títulos catalogados \\
\end{tabular}

\begin{tabular}{|c|c|}
\hline \multicolumn{2}{|c|}{ Grupo 3: Difusión de la información } \\
\hline $\begin{array}{l}\text { - Plantilla } \\
\text { - Tipos de acceso } \\
\text { - Cobertura (catálogos, bases de datos, etc.) } \\
\text { - Préstamos } \\
\text { - Número de cuestiones de referencia } \\
\text { - Uso de materiales en la biblioteca } \\
\text { - Préstamo interbibliotecario } \\
\text { - Respuestas verbales } \\
\text { - Reclamaciones } \\
\text { - Formación contínua del personal } \\
\text { - Formación de usuarios } \\
\text { - Porcentaje total de circulación }\end{array}$ & $\begin{array}{l}\text { - Peticiones satisfechas/no satisfechas } \\
\text { - Coste } \\
\text { - Pertinencia } \\
\text { - Eficiencia } \\
\text { - Efectividad } \\
\text { - Exhaustividad } \\
\text { - Precisión / relevancia temática y psicológica } \\
\text { - Circulación per cápita } \\
\text { - Porcentaje de circulación por tipo de usuario } \\
\text { - Porcentaje de circulación por materias } \\
\text { - Suministro electrónico de información } \\
\text { - Diferenciación en tipos de préstamo }\end{array}$ \\
\hline $\begin{array}{l}\text { Grupo 4: Extensión bibliotecaria y } \\
\text { animación sociocultural } \\
\text { - Bibliotecas sucursales } \\
\text { - Bibliotecas móviles } \\
\text { - Colaboración con otras entidades } \\
\text {-Dinamismo/estancamiento } \\
\text { - Tasa de actividad }\end{array}$ & $\begin{aligned} \text { Grupo 5: Evaluación: } \\
\text { - Coste del Proceso } \\
\text { - Consecución de objetivos } \\
\text { - Flexibilidad de la biblioteca } \\
\\
\text { - Formación continua } \\
\text { - Eficiencia } \\
\text { - Eficacia }\end{aligned}$ \\
\hline
\end{tabular}

Scire. 2 : 1 (en.-jun. 1996). 
1. Características mensurables:

- Tiempo

- Precio y costo

- Pruebas de campo y de prestación de servicios

- Errores

- Producción y procesos de elaboración

- Fiabilidad del equipo técnico o tecnológico
2. Efectos y condiciones observables

-Incompetencia

-Resultados negativos

-Intento fallido de satisfacer al usuario

-Fallo en el servicio.

3. Rasgos y actitudes de comportamiento observables

Estas características son positivas (calidad) o negativas (no-calidad).

-Características de buena calidad: Personal cortés, servicial, cuidadoso, educado, amable, accesible,

considerado, diligente, cooperador, acertado, honesto, fiable, comprensivo, interesado, cualificado, competente, etc.

-Características que demuestran mala calidad: Descortés, indiferente, descuidado, rudo, antagónico, arbitrario, lento, indeciso, desacertado, deshonesto, negativo, incompetente, no cualificado, etc.

\section{Cuadro 3: Indicadores para establecer las características de la calidad en los servicios bibliotecarios}

- Número de suscripciones

- Obsolescencia del material

- Presupuesto asignado

- Porcentaje total de adquisiciones

- Porcentaje de adquisiciones por materias

- Adquisiciones en formato electrónico

Los indicadores para establecer las características de la calidad en los servicios bibliotecarios pueden englobarse en tres grandes categorías: característi-

\begin{tabular}{|l|}
\hline \\
-Colaboración \\
-Compañerismo \\
-Comunicación horizontal \\
-Comunicación vertical \\
-Convicción en su trabajo \\
-Cooperación \\
-Creatividad \\
-Diversificación de tareas \\
-Grado de autonomia \\
-Motivación \\
-Originalidad \\
-Satisfacción del personal \\
\hline
\end{tabular}

Cuadro 4: Indicadores generales aplicables al personal de la biblioteca

Scire. $2: 1$ (en.-jun. 1996). 
cas mensurables, efectos y condiciones observables, y rasgos y actitudes de comportamiento observables.

Por último, estableceremos la distinción de indicadores generales aplicables al personal de la biblioteca.

Además, para efectuar los diagnósticos se utilizarán las siguientes herramientas (5): histogramas, gráficos, diagrama de Pareto, matriz de prioridades, diagrama de causa-efecto, organigramas, Brainstorming (tormenta de ideas), matriz de prioridades, tablas, etc.

12. Análisis y evaluación de las soluciones. Puesta en funcionamiento de las mismas. En esta fase se podrán hacer pronósticos y balances de previsión, además la corrección debe de estar presente en todo momento.

13. Seguimiento continuo de la evolución del programa de calidad.

Lectura para adultos

Préstamo para a dultos

Publicaciones periódicas

Infor mación bibliográfic a

Préstamo interbiblioteca rio

Reprografía

$\mathrm{N} / \mathrm{S}$

Ejemplo 1: Diagrama de barras de los servicios más demandados por los usuarios

Scire. $2: 1$ (en.-jun. 1996). 


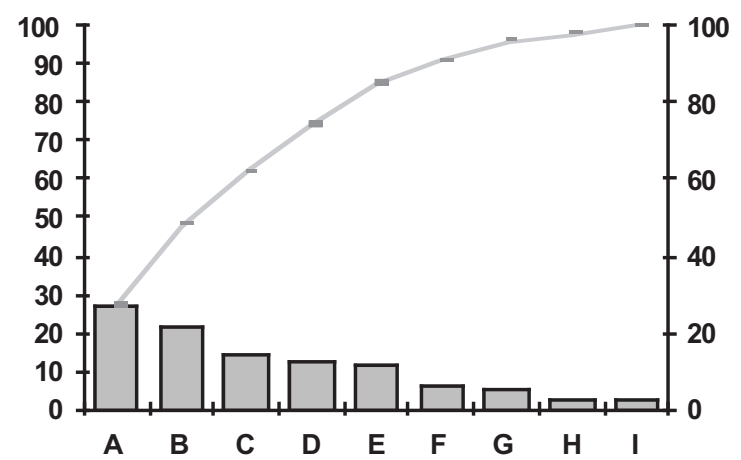

Ejemplo 2: Diagrama de Pareto. Representación I

Debilidades que presenta el centro desde el punto de vista del usuario

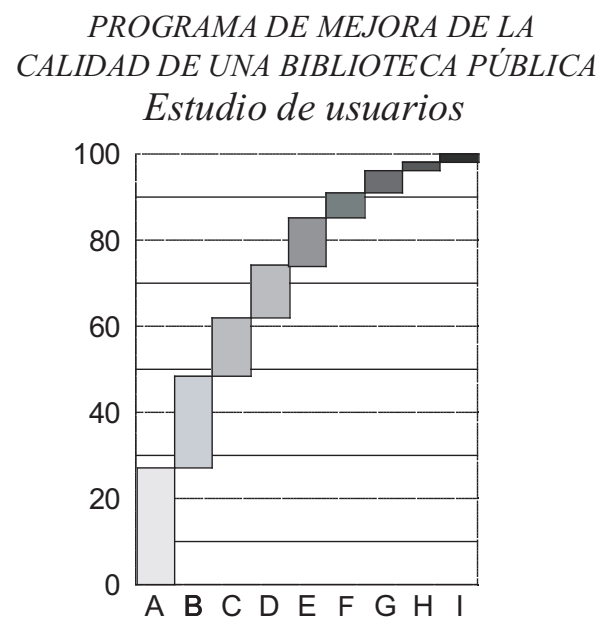

Diagrama de Pareto para la detección de problemas del servicio bibliotecario

Ejemplo 2: Diagrama de Pareto. Representación I

Scire. 2 : 1 (en.-jun. 1996). 


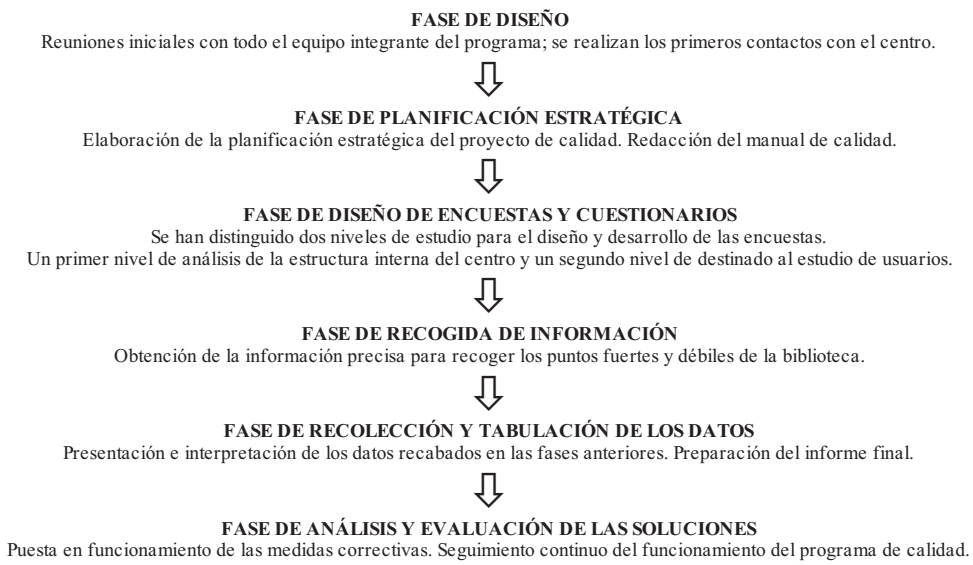

Fig. 2. FAses del modelo de calidad propuesto

\subsection{Resultados: debilidades y problemas que presentan las bibliotecas públicas}

A la hora de poner en marcha este modelo hemos de tener presentes las debilidades y problemas que plantean, de forma general, las bibliotecas públicas. Así, uno de los principales problemas de éstos centros es que persiguen muchos objetivos. Las bibliotecas podrían ser definidas como entidades con objetivos múltiples. En el fondo se trata de proveedores de servicios en el sector de la educación, la información, la cultura y el recreo u ocio para un círculo de clientes heterogéneo. Un análisis de los distintos mercados de las bibliotecas muestra que estos son demasiado amplios: además de los mercados horizontales (las bibliotecas están al servicio del público en general, de la economía, de la enseñanza, y de otros), están los mercados verticales (así, el mercado de "enseñanza" puede dividirse en varios segmentos entre el jardín de infancia y la universidad). Cada uno de estos mercados necesita distintos productos y servicios y resultaría muy pesado para una biblioteca ofrecer todo esto de tal suerte que se pueda conseguir un alto grado de satisfacción del conjunto de las demandas de los usuarios.

Las declaraciones sobre la función de la biblioteca tienden por naturaleza a un tono fuertemente moralizante y a formulaciones generales. Ello no quiere decir que se tengan que rechazar en bloque todas las expresiones sobre las funciones de la biblioteca, sino que se puede ganar más concentrándose en objetivos

Scire. 2 : 1 (en.-jun. 1996). 
próximos o lejanos alcanzables dentro de planteamientos más caseros. Por otra parte, las funciones que puede desarrollar una biblioteca pública no son única ni necesariamente las que estamos habituados a identificar como tales; pueden ser más amplias, más concretas o distintas. Es necesario decidir las que se deben crear, potenciar o suprimir de acuerdo con los estudios de mercado, los objetivos, las evaluaciones y la dinámica generada por el propio centro.

Hasta ahora las bibliotecas han tratado de satisfacer en la medida de lo posible la demanda de un mercado horizontal (no especialistas, estudiantes, comerciantes, niños, etc.), mientras que simultáneamente trataban de ofrecer materiales y servicios de distinto nivel (desde el elemental hasta el superior y más complicado) para cada uno de sus mercados verticales. A fin de cuentas las bibliotecas mantienen en pie una gama de productos muy ambiciosa. En cierto sentido la biblioteca trata de ser un "supermercado del saber". Pero dado que no puede cobrar por todos sus servicios (ni está en disposición de adoptar una postura abiertamente comercial o por lo menos de cubrir los costes en aquellos servicios en los que puede cobrar algo al usuario final), cada vez va a ser más difícil ofrecer servicios "de alto costo" o añadir nuevos servicios a los ya existentes.

Una segunda debilidad o problema al que se enfrentan estos centros bibliotecarios es su concepto mismo de público ¿Quién debe crear y sostener las bibliotecas públicas?. ¿Son éstas un monopolio estatal?. La biblioteca pública que históricamente nació de bibliotecas privadas o fue obra de pequeñas comunidades ha terminado por admitir y hasta exigir una mayor intervención de la Administración Central; no sólo por la necesidad de provocar la demanda social allí donde no existe, sino porque además ha ido aumentando la falta de autosuficiencia de la biblioteca y la necesidad que ésta tiene de órganos centrales de apoyo para poder cumplir con su misión. Pero con ello, se está también corriendo el peligro de que la biblioteca deje de ser "escuela de libertad".

Otro peligro es el de la burocratización de la biblioteca. Si ésta es algo ajeno a la comunidad, si el bibliotecario no es responsable ante la misma sino ante instancias superiores, si es antes el trabajo perfecto que las necesidades del posible usuario, es posible que la biblioteca, a pesar del esfuerzo económico que supone, no cumpla función alguna.

\subsection{Conclusiones: la figura del usuario, eje central de todo modelo de ges- tión de calidad total}

La gestión de la calidad pasa necesariamente por identificar las necesidades de los usuarios y determinar los indicadores que permitan evaluar los beneficios y resultados obtenidos como resultado del proceso del programa de calidad. Pero, ¿cómo se definen las necesidades?, ¿cómo identificarlas y definirlas?. ¿Tienen que ofrecer las bibliotecas a la población lo que le gustaría leer, o lo que necesi-

Scire. 2 : 1 (en.-jun. 1996). 
ta?. ¿Acaso lo que necesita con urgencia?... Es necesaria la distinción entre necesidad no conocida o no activa, necesidad no expresada y necesidad expresada. Del mismo modo podría distinguirse entre demanda, deseo y necesidad (Sanz Casado, 1994, p. 23-31).

Debemos considerar que en las necesidades de información de los usuarios se deben evaluar dos factores:

a) La eficiencia de los servicios, basándonos tanto en la accesibilidad como en la organización interna.

b) La eficacia de los rendimientos

No olvidemos, que el primer paso para disponer de un sistema de calidad bibliotecario es planificar la calidad y ello significa situarse en la perspectiva del usuario. Decidir desde el punto de vista de éste implica atender sus demandas. No se pueden tomar decisiones que tengan gran incidencia en los usuarios sin conocer lo que realmente piensan ellos. Es importante tener presente toda la información subjetiva y suficientemente representativa expresada espontáneamente por los usuarios. Hemos de tener presente que la calidad de los servicios está vinculada a la satisfacción de las necesidades de los usuarios. De tal forma hemos de

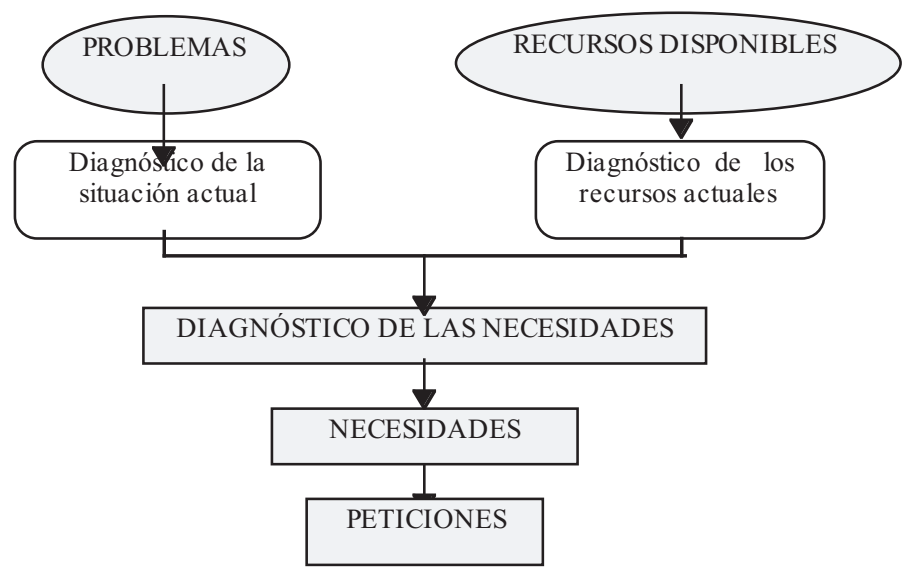

Figura 3. Necesidades del usuario

Scire. $2: 1$ (en.-jun. 1996). 
sumar la información obtenida directamente de los usuarios y contrastarla con la obtenida, tradicionalmente, por la propia organización a través de su personal, y así podremos conocer mejor cuáles son los problemas y las necesidades de los usuarios-clientes de los centros bibliotecarios.

En el ámbito de la percepción del usuario es perfectamente aplicable el esquema según el cual las necesidades de los usuarios serían el resultado de los problemas percibidos por los usuarios en función de los recursos existentes para solucionarlos (vid. figura 3) . Los usuarios perciben un hecho o circunstancia como problema cuando tienen noticia de que existen recursos disponibles, que ellos creen adecuados para solucionarlos, y con esta información se forman un diagnóstico de las necesidades que ellos tienen y exigen la aplicación de recursos para atenderlas.

Los problemas percibidos por los usuarios y el personal de la biblioteca no tienen porqué coincidir y, de hecho, normalmente, nunca sucede así. Los problemas del personal bibliotecario quedan aislados de las vivencias y percepciones de sus usuarios. También, es frecuente que la percepción de un problema no sea la misma para diferentes grupos o segmentos de usuarios con intereses diferentes. En definitiva, lo que se puede observar, es que la percepción de un problema es claramente una cuestión profundamente subjetiva de muy difícil objetivación. Por ello, hemos de prestar más atención a los estudios sobre las necesidades sociales, para poder conocer así mejor los problemas y los recursos existentes.

Para conocer las necesidades y demandas de los usuarios existen dos caminos complementarios muy útiles (6):

- Identificación externa: Se trata de descubrir estas necesidades mediante estudios de mercado científico adaptados al entorno bibliotecario, encuestas a los usuarios, estudios sobre cómo se gestiona la calidad en otros centros bibliotecarios, etc. Otros métodos son la creación de espacios participativos desde los cuales se pueden detectar las necesidades de los usuarios de estos servicios o recabar directamente las opiniones de las personas que acuden a estos centros informativos. Asimismo, existen diversos recursos para conocer cuál es el grado de satisfacción de los ciudadanos y para averiguar cuáles son sus necesidades no cubiertas o cómo éstas evolucionan a lo largo del tiempo.

- Identificación interna: Las personas prestadoras de los diferentes servicios bibliotecarios y, en concreto, el personal de atención directa al público pueden aportar informaciones útiles para conocer cuáles son las necesidades o las demandas de los usuarios de los mismos y el grado de satisfacción por los servicios recibidos. Otra fuente de información es el análisis de las sugerencias y la quejas realizadas por los propios usuarios de los servicios

Scire. 2 : 1 (en.-jun. 1996). 
bibliotecarios. Las sugerencias y las quejas son un mecanismo valioso para saber por dónde empezar a mejorar la gestión de los servicios de la biblioteca.

Mediante el estudio de los usuarios, obtenemos información sobre sus actitudes, preferencias, valoraciones y sugerencias de mejora. Recogemos datos sobre cada una de las siguientes situaciones (grupos):

1. Por qué los usuarios, sintiéndose insatisfechos, no se quejan.

2. Por qué algunos usuarios se dan ya por perdidos y qué es necesario para recuperarlos.

3. Por qué algunos usuarios son indiferentes y qué se puede hacer respecto a ello.

4. Por qué algunos usuarios están satisfechos.

5. Por qué algunos usuarios están muy contentos.

6. Por qué los usuarios siguen volviendo; los usuarios fieles, los habituales.

Al mantenerse un estrecho contacto con estos seis grupos, así como con un séptimo grupo -los usuarios que presentan quejas-, la biblioteca tiene una idea sobre el papel de los usuarios o mejor dicho, y hablando en términos de calidad, de cómo los usuarios están reaccionando a las diferentes características de la calidad de los servicios y qué acciones han de llevarse a cabo para mejorar y mantener la calidad.

Además existe un octavo grupo: los que no son usuarios o clientes, que son usuarios o clientes potenciales, pero que también han de ser estudiados para determinar por qué no están usando un determinado servicio. Éste es el reto de los estudios de mercado; estudios que han de ser dirigidos como parte integral de un programa cliente-mercado continuo y extenso.

Para un estudio de usuarios es necesaria la cuantificación y tipificación del usuario de estos sistemas de información. Así pues, nuestro propuesta comenzará por una identificación y cuantificación de los usuarios de los distintos servicios ofertados por la biblioteca. Se enviará una encuesta a la biblioteca que sirva para caracterizarla. En la encuesta se detectarán, por una parte, los recursos del centro: personal, equipos, fondos documentales y tratamiento de los mismos; y por otra las características generales de la información requerida por ellos: tipos de documentos primarios, fuentes de referencia, etc. La encuesta también habrá de reflejar los servicios que necesita la institución encuestada. A continuación se estudiará la oferta de productos: índices bibliográficos impresos, bases de datos automatizadas, etc. Se finalizará con un balance de la adecuación de la oferta y la demanda, y con una exposición de propuestas de actuación.

Entendida la biblioteca como servicio público, interesa analizar en qué medi-

Scire. 2 : 1 (en.-jun. 1996). 
da las prestaciones que se ofertan al usuario están en consonancia con la demanda. Y a la inversa, cómo adecuar a las demandas los productos. Para ello, se utilizan una serie de técnicas o herramientas, entre las que destacan los estudios de mercado o marketing y los despliegues de función de la calidad (QFD).

Anteriormente hemos mencionado la utilidad que pueden tener los estudios de mercado para conocer las necesidades de los usuarios y así planificar mejor los ámbitos de actuación de la biblioteca. A pesar de la gran utilidad de estos estudios, el personal bibliotecario, y en concreto el director del centro, no puede basar su política de actuación únicamente en las encuestas o en los estudios científicos de mercado. Deben llegar más lejos, para no alejar al centro de la sociedad; una sociedad que sufre continuas y rápidas transformaciones. Por ello debe tener la capacidad de imaginar y de anticipar el futuro. Con los estudios de usuarios se analiza el presente (saber), pero un buen profesional ha de utilizar estos estudios y sus conocimientos para predecir el mañana (prever) y estar preparado para (poder) las nuevas necesidades y demandas que exigirán los usuarios en un futuro (7).

\section{Notas}

(1) Calidad total es un principio, una filosofía productiva según la cual el consumidor, el usuario, aquel que accede a un producto o servicio es el auténtico soberano de todo el proceso de producción y cobertura de un servicio. Todo ello se ha de hacer en función de la demanda del mercado, en la forma y función exigida y en el momento solicitado. Para ello, es necesaria la implantación de sistemas de calidad total que van a dejar que sea el usuario o cliente quien defina qué quiere, cuándo y cómo lo quiere, y será entonces cuando comenzaremos a trabajar. Pondremos todo el esfuerzo y energías en satisfacer a nuestros usuarios, pero ahorraremos una inmensa, a veces incalculable, energía (y costes) en elaborar y mantener bienes y servicios cuya dudosa sintonía con los gustos del usuario obliga a grandes esfuerzos a la hora de difundir y poner a disposición los productos y ofertas de nuestro centro de información.

(2) Pinto Molina, 1992, p.240. Cita a Cleveland y Cleveland, 1983, p. 112-119.

(3) Concepto de indicador: Se denomina indicador a la variable que hace referencia a un hecho y es susceptible de alguna clase de medición. Es decir, un indicador es el resultado de cuantificar las distintas actividades realizadas por los servicios bibliotecarios, y su finalidad no es otra que contribuir a determinar en qué grado se han conseguido los objetivos previstos.

No obstante, el concepto de indicador varía de unos autores a otros. Para unos cumple una función informativa, para otros es un instrumento en la toma de decisiones, o bien cumple una función de evaluación. Sin embargo, hay plena coincidencia en que el indicador debe tomar valores numéricos, independientemente de la forma que adopte, y que puede referirse a la situación existente en un periodo de tiempo concreto o a su evolución en el tiempo.

La ventaja del uso de indicadores sobre cualquier otro método es que traducen la rea-

Scire. $2: 1$ (en.-jun. 1996). 
lidad en cifras cuya interpretación no se presta a discusión, y pueden ser un instrumento de primer orden en la planificación de los servicios bibliotecarios, en el control de los mismos, en la predicción de sus efectos a medio y largo plazo o en el impacto de una determinada política bibliotecaria sobre los usuarios.

(4) El servicio de extensión cultural, por sus característicasa específicas, se concibe como un grupo diferente al de difusión de la información a la hora de delimitar los grupos de indicadores básicos.

(5) Incluímos una serie de ejemplos extraídos de los resultados de una aplicación de un programa de calidad realizado a una biblioteca pública (Ruiz Abellán et al, 1996).

(6) Adaptación a nuestro modelo de las aportaciones teóricas recogidas en la obra de López Camps y Gadea Carrera (1995, p. 94-103).

(7) Tal es el lema comtiano: "Saber para Prever, con el fin de Poder". Vid. Izquierdo (1995, p. 7, nota 26), Espinosa e Izquierdo (1994, p. 21), Sagredo e Izquierdo (1983, p. 323, 372, tabla, col. 2).

\section{Referencias Bibliograficas}

Akao, Yoji (ed. lit). Despliegue de funciones de calidad : QFD : integración de necesidades del cliente en el diseño del producto. Madrid : Raimundo Fernández Villaverde, 1993.

Blake, R. R. ; Mouton, J. S. : The Managerial Grid. Houston : Gulf, 1964.

Bockman, J.R. : Quality assurance (QA) and the management of information services. // Journal of Information Science. (1991) 127-135

Butterwick, N.B. Total Quality Management in the university library. // Library Management. 14 : 5 (1993) 23-25.

Clavel, J. P. ; (et al.). L'évaluation des bibliothéques universitaires. Montreal : UPELF, 1984.

De Prospo, E. ; Altman, E. ; Beasley, K. Performance Measures for Public Libraries. Chicago : American Library Association, 1973.

Ellis, D. ; Norton, B. Implementing BD5750/ISO 9000 in libraries. London : ASLIB, 1993.

Espinosa, M-B; Izquierdo Arroyo, J.M. ; et al. Tecnologías documentales : Memorias ópticas. Madrid : Tecnidoc, 1994.

Horts, E. ; Hans-Dieter, W. Bibliotecas Públicas hoy y mañana : nuevos planteamientos de objetivos y gestión: Coloquio Internacional organizado por la Fundación Bertelsmann. Salamanca ; Madrid : FGSR ; Pirámide, 1987. (Biblioteca del Libro. Serie Minor).

Izquierdo Arroyo, J. M. La Organización documental del conocimiento : I/1 : El marco documental. Madrid : Tecnidoc, 1995.

Johansen, C.G. The use of quality control principles and methods in library and information science theory and practice. // Libri. 42 (1992) 283-295.

Juran, J.M. ; Gryna, F. M. Manual de control de calidad. 4ª ed. Madrid : McGraw Hill, 1993. 2 vol.

Scire. 2 : 1 (en.-jun. 1996). 
Jurow, S. ; Barnad, S. Integrating Total Quality in a Libray setting. New Jork, etc. : The Haworth Press, 1993.

KING research Ltd. Keys to success : performance indicators for publics libraries. London

: Office or Arts and Libraries, 1990. (Library Information series $n^{\circ} 18$ ).

Lancaster, F. W. He measurement and evaluation of library services. Washignton : Information Resources Press, 1977.

Lancaster, F. : The Measurement and Evaluation of Library Services. $2^{\circ}$ ed. México : UNAM, 1991.

Lininger, Ch. ; Warwick, D. : La encuesta por muestreo : teoría y práctica. México : CECSA, 1985.

López Camps, J. ; Gadea Carrera, A. Servir al ciudadano : Gestión de la calidad en la Administración Pública. Barcelona : Ed. Gestión 2000, 1995.

Michel, J. Pratique du management de l'information : analyse de la valeur et resolution de problémes. París : ADBS, 1992.

Moore, N. Medición de la eficacia de las bibliotecas públicas. París : UNESCO, 1989.

Pagaza García, R. Manual para obtener indicadores como apoyo a la evaluación de servicios bibliotecarios en instituciones de Educación Superior. México : UNAM; ANUIES, 1989.

Riggs, D. E. Strategic Quality Management in Libraries. // Advances en Librarianship. (1992) 93-105.

Rosander, A. C. La búsqueda de calidad en los servicios. Madrid : Díaz de Santos, 1992.

Ruiz Abellán, J.; Izquierdo Alonso, M.; Leyva Cuesta, M.J.; Moreno Fernández, L.M. Aplicación de un programa de calidad a la Biblioteca Pública de Almería. // V Jornadas Españolas de Documentación Automatizada. Cáceres : ADABMEX; FESABID, 1996. p. 389-395.

Sagredo, F. ; Izquiero Arroyo, J. M. Concepción lógico-lingüística de la Documentación. Madrid : Ibercom, 1983.

Sanz Casado, E. Manual de estudios de usuarios. Madrid : FGSR; Madrid : Pirámide, 1994.

Saracevic, T. ; et al. : Causes and dynamics of user frustration in and academic library. // College and Research libraries. 38 : 1 (1977) 7-18.

Senlle, a. \& Stoll, G. Calidad total y normalización. ISO 9000 : las normas para la calidad en la práctica. Barcelona : Gestión 2000, 1994.

Sutter, E. Services d'information et qualité : comment satisfaire les utilisateurs. París : ADBS, 1992.

Totterdell, B. ; Bird, J. The effective library : report of the Hillingdon project on public library effectiveness. London : Library Association, 1976.

Underwood, P. G. : Managing change in libraries and information services. London : Clive Bingley, 1990.

UNISIST. Directrices para los estudios relativos a los usarios de la información : Versión experimental. París : UNESCO, 1981. (PGI. 81/WS/2).

Walker, D. El cliente es lo primero : estrategia para un servicio de calidad. Madrid : Díaz

Scire. 2 : 1 (en.-jun. 1996). 
de Santos, 1991.

Wintehall, T. Quality in library and information services : a review. // Library management, $14: 4$ (1992), 4-12.

Yan House, N. ; et al. Output measure for public libraries. $2^{\mathrm{a}}$ ed. Chicago : American Library Association, 1987.

Scire. 2 : 1 (en.-jun. 1996). 